\title{
Commentary
}

\section{The case for more comprehensive data on household food insecurity}

\author{
Valerie Tarasuk, PhD; Timmie Li, BSc; Andrew Mitchell, MA; Naomi Dachner, MSc
}

Tweet this article

\section{Introduction}

Household food insecurity refers to the inadequate or insecure access to sufficient food because of financial constraints. The inclusion of questions related to food insecurity on national population health surveys over the past two decades has yielded a solid understanding of the social epidemiology of food insecurity in Canada and established it as an indicator of nutrition inequity and a potent social determinant of health. There is also growing recognition that problems of household food insecurity cannot be managed by community responses. Policy interventions are required to address the conditions underpinning this vulnerability. Awareness of the importance of food insecurity has heightened the demand for information about the extent of this problem.

It is increasingly common for food insecurity statistics to be included in status reports on the health and well-being of the population at the provincial, municipal, and regional levels. Such reporting is important in enabling policymakers, social planning agencies, public health authorities, and other stakeholders to gauge the extent of deprivation in their communities, monitor the impact of changing social and economic conditions on prevalence, and set priorities for interventions. However, the limited quality of publicly available data on household food insecurity impedes such data usage efforts. The purpose of this commentary is to provide an overview of the monitoring of household food insecurity in Canada, describe the statistics on household food insecurity of most relevance to population health, and identify critical gaps in the current reporting of the prevalence of food insecurity.

\section{Food insecurity measurement in Canada}

Questions about child hunger and household food insufficiency began to appear on national population health surveys in 1994, but systematic assessment of household food insecurity did not begin until 2004 when the Household Food Security Survey Module (HFSSM), a standardized, validated, 18-item scale of severity developed by the U.S. Department of Agriculture, was added to Cycle 2.2 of the Canadian Community Health Survey (CCHS). ${ }^{1}$ The HFSSM is designed to characterize the level of food insecurity in the household overall. The questions capture experiences of food insecurity ranging from concerns about running out of food before there is more money to buy more, to the inability to afford a balanced diet, to going hungry, missing meals, and in extreme cases, not eating for a whole day because of a lack of food and money for food. Ten questions refer to household conditions generally and adults' experiences in particular, and for households that include members under 18 years of age, an additional eight questions are asked to assess the children's experiences of food insecurity.

Health Canada undertook an extensive examination of the survey results from CCHS 2.2 to confirm the appropriateness of the HFSSM for food insecurity assessment in Canada. ${ }^{1}$ They also developed a classification scheme to define moderate and severe food insecurity ${ }^{1}$ and proposed classifying any household with zero or one affirmative response on either the adult or child-referenced scale as food secure. Since that time, a substantial body of research has emerged, indicating that even a single affirmative response on the

\section{Highlights}

- As awareness of the population health importance of household food insecurity and its relation to public policy decisions grows, interest in food insecurity statistics is burgeoning at the provincial, municipal, and regional levels.

- The publicly available data on its prevalence are insufficient to support stakeholders' interests and needs for timely, locally relevant information.

- Several jurisdictions fail to measure food insecurity when its inclusion on the Canadian Community Health Survey is optional.

- We recommend mandatory measurement annually, with routine reporting of the total number of persons living in food insecure households and marginal food insecurity counted as part of food insecurity.

HFSSM denotes significant vulnerability, with important consequences for individuals' health and well-being. These findings make a strong case for treating marginally food insecure households as a discrete group and including them in estimates of the population prevalence of household food insecurity, but to date Health Canada's classification scheme has not been revised. Because this scheme defines the reporting practices of Statistics Canada and most provincial and regional authorities, updating it to reflect current science will improve food insecurity assessment nationally. 


\section{Monitoring the prevalence of household food insecurity}

Food insecurity is monitored on the annual cycles of CCHS. The survey sample is designed to be representative of $98 \%$ of the Canadian population aged 12 and over, omitting individuals on First Nation reserves, in institutions, in the Canadian Armed forces, and in some remote areas. The application of household weights, developed by Statistics Canada to account for the sampling design and patterns of nonresponse, yields a population-representative sample of Canadian households for each survey year, enabling estimation of the proportion and number of households who reported food insecurity over that year. While these weights can be used to generate national and provincial/territorial prevalence estimates, they have not been calibrated for use at sub-provincial/ territorial levels, limiting the determination of food insecurity prevalence at the regional or community level.

The national prevalence of food insecurity in 2007-08 and 2011-12, the two cycles for which nationally representative data are available, is presented in Table 1. Irrespective of whether marginally food insecure households are counted as food insecure, the prevalence has risen significantly over this period. Given the abundance of evidence that food insecurity is detrimental to health, this increase should be cause for serious concern.

\section{Estimating the number of individuals living in food insecure households}

In addition to tracking the household prevalence, it is critical to track the number of people exposed to household food insecurity because this exposure is a highly significant predictor of individuals' health and well-being. Tracking the number or proportion of people (versus households) affected by food insecurity is also consistent with the way in which other population statistics are reported (e.g. the rate of child poverty, the unemployment rate, the number of people using food banks), and perhaps because of this, it appears to be the preferred metric for reporting on household food insecurity in lay publications and the status reports issued by various social planning agencies, public health authorities, and other stakeholders groups. Accessing such information is complicated, however, because the
CCHS sample only comprises persons aged 12 and over.

Through the application of person weights, population-representative estimates of the number of individuals 12 years of age and older living in food insecure households can easily be derived from CCHS data, but this seriously understates the total number of persons affected and renders invisible the heightened vulnerability of younger children to household food insecurity. The total number of persons living in food insecure households can be estimated by applying household weights to the data on household composition in CCHS. Using this approach, we find that 3939500 Canadians lived in food insecure households in 2011-12; that is 529300 more than the number in 200708 (Table 1). Moreover, one in five people living in a food insecure household in these years was a child under 12 years of age.

The variables required to estimate household prevalence and the total number of persons living in food insecure households are contained in the CCHS Master Files available to researchers in the Statistics Canada Research Data Centres and the Share Files available to the provincial and territorial governments, but they are not available in the public use files for CCHS. Thus, this information is inaccessible to agencies and organizations without the capacity and authorization to analyze the CCHS microdata.

\section{The frequency of food insecurity measurement}

The HFSSM has been included as core content on the 2007-08, 2011-12, and 201718 cycles of CCHS, but in the intervening years, the decision to administer the module has rested with the provincial and territorial governments. Only Alberta, Quebec, Nova Scotia, Northwest Territories, and Nunavut have elected to measure household food insecurity on every cycle since the HFSSM was introduced in 2005. Yet, food insecurity prevalence rates across Canada are not static, as indicated by the significant increase in prevalence nationally

TABLE 1

Number and proportion of households reporting food insecurity and number of people living in food insecure households in Canada, 2007-08 and 2011-12

\begin{tabular}{|c|c|c|}
\hline & 2007-08 & 2011-12 \\
\hline \multicolumn{3}{|l|}{ All households } \\
\hline Marginal food insecurity & $446000(3.6 \%)$ & $536200(4.1 \%)$ \\
\hline Moderate food insecurity & $626300(5.1 \%)$ & $759600(5.8 \%)$ \\
\hline Severe food insecurity & 329500 (2.7\%) & $333500(2.5 \%)$ \\
\hline Total & $1401800(11.3 \%)^{\mathrm{a}}$ & $1629400(12.4 \%)^{\mathrm{a}}$ \\
\hline \multicolumn{3}{|l|}{ All persons } \\
\hline Marginal food insecurity & 1155100 & 1389800 \\
\hline Moderate food insecurity & 1570800 & 1897600 \\
\hline Severe food insecurity & 684200 & 652000 \\
\hline Total & 3410200 & 3939500 \\
\hline \multicolumn{3}{|c|}{ All persons, 12 years and older } \\
\hline Marginal food insecurity & 932700 & 1107100 \\
\hline Moderate food insecurity & 1237200 & 1518700 \\
\hline Severe food insecurity & 556300 & 537300 \\
\hline Total & 2726300 & 3163000 \\
\hline \multicolumn{3}{|l|}{ All persons, under 12 years } \\
\hline Marginal food insecurity & 222400 & 282800 \\
\hline Moderate food insecurity & 333500 & 378900 \\
\hline Severe food insecurity & 127900 & 114800 \\
\hline Total & 683800 & 776500 \\
\hline
\end{tabular}

Note: Totals may not add up due to rounding.

${ }^{a}$ Chi-square test of proportions indicates significant difference, $\mathrm{p}<0.0001$. When marginal food insecurity is omitted from the total prevalence of food insecurity, the chi-square test of difference between cycles is significant at $p=0.0017$. 
between 2007-08 and 2011-12. Food insecurity rates vary in response to changes in macroeconomic conditions ${ }^{2,3}$ and policy decisions that impact household finances..$^{3-6}$

Provincial and territorial governments' decisions to opt out of food insecurity measurement impede the use of data by policymakers, public health authorities, and other stakeholders within these jurisdictions to assess the extent of deprivation in their areas and set evidence-based priorities for program and policy responses. Gaps in measurement also thwart research and evaluation activities to identify the effects of specific policy decisions on food insecurity prevalence and severity. Only by being able to compare prevalence rates before and after particular policy reforms have we come to understand the power of federal and provincial policy decisions to impact this problem. ${ }^{3-6}$ Without pre- and post-intervention data, how can we possibly evolve our understanding of 'what works'? The sporadic measurement of food insecurity is retarding the development of the knowledge needed to devise evidence-based policy responses to a serious, widespread and growing population health problem.

\section{The availability of information on food insecurity prevalence}

CANSIM tables provide online access to estimates of the prevalence of moderate and severe household food insecurity combined $^{7}$ and the prevalence of individuals 12 years of age and older living in moderately or severely food insecure households for 2007-08 and 2011-12.8 These estimates are available at the national, provincial, and territorial level, with sub-provincial estimates available for the person-weighted calculations of exposure of individuals aged 12 and older. Health Canada has posted summary statistics on moderate and severe food insecurity in the provinces and territories that participated in measurement in 2005 and 2009-10, and in August 2017, they posted charts including provincial and territorial prevalence estimates for 2013-14.

Some provincial and territorial governments and some local health authorities have published reports on food insecurity rates for their jurisdictions for years other than those covered by CANSIM, but this reporting typically mirrors Statistics Canada's CANSIM releases. With very few exceptions, rates of moderate and severe food insecurity have been combined, marginally food insecure households have been counted as food secure, and individual exposure has only been reported for people 12 years of age and older. Additionally, it is not clear that any jurisdiction has committed to the regular, timely reporting of food insecurity statistics.

As part of the knowledge translation strategy of our CIHR-funded research program, 'PROOF', we produced more comprehensive reports on household food insecurity prevalence in Canada in 2011, 2012, 2013, and 2014 , e.g. ${ }^{9}$. The overwhelming interest in these reports suggests that they have filled an important void for stakeholders and the public at large. However, knowledge translation work undertaken as part of a time-limited research initiative cannot substitute for the regular, timely release of statistics on food insecurity on government websites.

\section{Conclusions and recommendations}

As knowledge of the health and social consequences of household food insecurity in Canada grows and the intersections of this problem with public policy decisions become better understood, the importance of food insecurity monitoring is becoming more evident. The only nationally representative measures we have indicate a substantial increase in the number of Canadians living in food insecure households between 2007-08 and 2011-12, highlighting the need for even more vigilance in the tracking of this problem going forward.

Understanding the scale of household food insecurity in our population hinges on tracking the proportion of households who are food insecure and the number of individuals living in food insecure households. Yet, the public reporting of exposure is currently limited to the number of individuals over the age of 12 who live in moderately and severely food insecure households, no information is available on the prevalence of food insecure households at the regional or municipal level, and marginally food insecure households continue to be treated as if they were food secure. Moreover, the treatment of food insecurity measurement as optional content on most cycles of CCHS precludes the use of evidence to inform actions in jurisdictions that choose not to measure the problem and impedes the research necessary to develop effective responses to food insecurity nationally and at the provincial/territorial level.

To address the limitations in data collection and reporting identified here, we recommend that

i. the routine reporting of food insecurity statistics in CANSIM tables be updated to include information on the total number of persons living in food insecure households;

ii. marginal food insecurity be recognized as food insecurity and included in calculations of prevalence;

iii. where possible, household weights for CCHS be calibrated at sub-provincial/ territorial levels so that more local level data on food insecurity can be made available; and

iv. the assessment of household food insecurity become core content on all cycles of CCHS.

\section{Acknowledgements}

This research was supported by a Programmatic Grant in Health and Health Equity from the Canadian Institutes of Health Research (CIHR) (FRN 115208). The authors are indebted to Andrée-Anne Fafard St-Germain for her critical comments on earlier drafts of this manuscript.

\section{Conflicts of interest}

The authors have no conflicts of interest to disclose.

\section{Statement}

The content and views expressed in this article are those of the authors and do not necessarily reflect those of the Government of Canada.

\section{References}

1. Health Canada. Canadian Community Health Survey, Cycle 2.2, Nutrition (2004) - Income-Related Household Food Security in Canada. Ottawa (ON): Office of Nutrition Policy and Promotion, Health Products and Food Branch, Health Canada; 2007. Report No.: 4696. 
2. Sriram U, Tarasuk V. Changes in household food insecurity rates among Census metropolitan areas from 2007 to 2012. Can J Public Health. 2015;106(5):e322-e7.

3. Emery J, Bartoo A, Matheson J, Ferrer A, Kirkpatrick S, Tarasuk V, et al. Evidence of the association between household food insecurity and heating cost inflation in Canada, 19982001. Canadian Public Policy. 2012; 38(2):181-215.

4. Loopstra R, Dachner N, Tarasuk V. An exploration of the unprecedented decline in the prevalence of household food insecurity in Newfoundland and Labrador, 2007-2012. Canadian Public Policy. 2015;41(3):191-206.

5. Li N, Dachner N, Tarasuk V. The impact of changes in social policies on household food insecurity in British Columbia, 2005-2012. Preventive Medicine. 2016;93:151-8.

6. Ionescu-Ittu R, Glymour M, Kaufman J. A difference-in-difference approach to estimate the effect of income-supplementation on food insecurity. Prev Med. 2015;70:108-16.

7. Statistics Canada. CANSIM database: Table 105-0546 - Household food insecurity measures, by presence of children in the household, Canada, provinces and territories, occasional (number unless otherwise noted) [Internet]. Ottawa (ON): Statistics Canada; 2013 [updated 2013, Dec 11; cited 2017 Apr 28]. Available from: http://www5.statcan.gc.ca/cansim/a26 ?lang $=$ eng\&id $=1050546$

8. Statistics Canada. Table 105-0547. Household food insecurity, by age group and sex, Canada, provinces, territories, health regions (2013 boundaries) and peer groups, occasional (number unless otherwise noted) [Internet]. Ottawa (ON); 2013 [updated 2013 Dec 11; cited 2017 Apr 28]. Available from: http://www5 .statcan.gc.ca/cansim/a26?lang = eng \&id $=1050547$

9. Tarasuk V, Mitchell A, Dachner N. Household food insecurity in Canada, 2012. Toronto (ON): Research to Identify Policy Options to Reduce Food Insecurity (PROOF); 2014. 